\title{
Proof of Character-Valued Index Theorems ${ }^{\star}$
}

\author{
Mark W. Goodman ${ }^{\dagger}$ \\ Joseph Henry Laboratories, Princeton University, Princeton, NJ 08544, USA
}

\begin{abstract}
A character-valued index is a generalization of the ordinary Dirac index to manifolds with nontrivial automorphism groups. A simple proof of the corresponding fixed-point theorem is presented which uses the techniques of supersymmetric quantum mechanics. This theorem relates the character-valued index to a topological integral of curvature forms on the fixed-point space of the automorphism in question.
\end{abstract}

\section{Introduction}

In recent years, topological methods have come to play an increasingly central role in theoretical physics, particularly in models with more than four spacetime dimensions. One of the most remarkable applications of topology to field theory is the Atiyah-Singer index theorem for the Dirac operator, [1] which describes the chiral content of the fermionic zero-modes in a particular background field configuration, containing both gravitational and gauge fields. This theorem contributed to an understanding of anomalies [2] and in particular how instantons can lead to the breaking of anomalous global symmetries [3]. Index theorems for families of field configurations [4] have improved our understanding of anomalous gauge symmetries.

The formal structure of topological indices is familiar to physicists in the form of Witten's supersymmetry index [5] $\operatorname{Tr}(-1)^{F}$. It measures the difference in the number of bosonic and fermionic states of zero energy, and, if it is not zero, indicates that supersymmetry cannot be spontaneously broken. Many authors [6] have noted the correspondence between the Witten index for supersymmetric quantum mechanics models and the Dirac index. This correspondence leads to a simple way of deriving the Atiyah-Singer index theorem and other related results.

Index theorems are particularly important in models in more than four spacetime dimensions. The spacetime then takes the form $M=M^{4} \times K$, where $M^{4}$ is

* Supported in part by NSF grant PHY-80/19754

+ Address after August 1, 1986: Institute for Theoretical Physics, University of California, Santa Barbara, CA 93106 USA 
four-dimensional Minkowski spacetime and $K$ is a compact internal space. The index of the wave operator on a particular type of tensor field on $K$ then determines the number of corresponding massless fields. The character-valued index is a generalization of the Atiyah-Singer index to the case where $K$ has a nontrivial automorphism group $G$ [7]. In the context of higher-dimensional theories, the corresponding index theorem will determine how the massless fields transform under $G$. This was discussed for continuous symmetries in [8] and this paper generalizes that analysis to the case of discrete groups $G$.

\section{The Character-Valued Index}

All topological indices contain a number of features in common, and it is useful to establish a general formal framework. On a particular Hilbert space $V$, there is a hermitian operator $Q$ whose square

$$
Q^{2}=\Delta
$$

is positive semi-definite. Usually, $V$ is the space of tensor fields on a manifold $K$ and $\Delta$ is the Laplacian for those fields. In addition, there is an operator $\Gamma$ which anticommutes with $Q$,

$$
\Gamma Q=-Q \Gamma
$$

and whose square is the identity $\Gamma^{2}=1 . \Gamma$ has eigenvalues \pm 1 , and separates the Hilbert space $V$ into two subspaces $V_{ \pm}$defined by

$$
\Gamma V_{ \pm}= \pm V_{ \pm} \text {. }
$$

By (2.2), $Q$ maps these subspaces into each other $Q: V_{+} \leftrightarrow V_{-}$, and therefore splits into two pieces $Q_{ \pm}$which act on the subspaces $V_{ \pm}$which act on the subspaces $V_{ \pm}$ respectively:

$$
Q_{+}: V_{+} \mapsto V_{-}, \quad Q_{-}: V_{-} \mapsto V_{+} .
$$

These two operators are formally hermitian conjugates $Q_{+}=Q_{-}^{*}$, up to questions of whether the domain of one coincides with the range of the other. Noting that $Q_{+}^{2}=Q_{-}^{2}=0$ and $Q_{+} Q_{-}+Q_{-} Q_{+}=\Delta$, we find that $Q_{ \pm} \Delta=\Delta Q_{ \pm}$, i.e. $Q_{ \pm}$preserves the eigenvalues of $\Delta$.

Vectors $\mathbf{v}$ in $V$ with positive (i.e. nonzero) eigenvalues of $\Delta$ are paired by the action of $Q_{+}$; for every such vector $\mathbf{v}_{+}$satisfying $\Gamma \mathbf{v}_{+}=+\mathbf{v}_{+}$and $\Delta \mathbf{v}_{+}=\lambda \mathbf{v}_{+} \neq 0$ the vector $\mathbf{v}_{-}=\lambda^{-1 / 2} Q_{+} \mathbf{v}_{+}$is its uniquely defined partner. For zero-modes of $\Delta$, this argument breaks down. If $\Delta \mathbf{v}_{+}=Q^{2} \mathbf{v}_{+}=0$, then we must have $Q_{+} \mathbf{v}_{+}=0$. The zeromodes of $\Delta$ split into two types, those in $V_{+}$and those in $V_{-}$, or more precisely those in $\operatorname{ker}\left(Q_{+}\right)$and those in $\operatorname{ker}\left(Q_{-}\right)$, where $\operatorname{ker}\left(Q_{+}\right)$denotes the kernel of the operator $Q_{ \pm}$, the subspace of $V_{ \pm}$which $Q_{ \pm}$maps to zero. The index of $Q$ is simply the difference in the dimensions of these two vector spaces

$$
I(Q)=\operatorname{dim} \operatorname{ker}\left(Q_{+}\right)-\operatorname{dim} \operatorname{ker}\left(Q_{-}\right) .
$$

This situation is formally identical to the algebra of supersymmetric quantum mechanics, where $Q$ is the supersymmetry charge, $Q^{2}$ is the hamiltonian, and 
$\Gamma=(-1)^{F}$ is the operator which counts fermion number modulo two. The defining property of $(-1)^{F}$ is that it anticommutes with all fermionic operators, including the supersymmetry charge.

Familiar examples include the Dirac index and the Euler character. For the Dirac index, $V$ is the space of Dirac spinors on a Riemannian manifold $M$ of dimension $d=2 k, Q$ is the Dirac operator $D$, and $\Gamma$ is the chirality operator $i^{k} \gamma_{1} \gamma_{2} \ldots \gamma_{d}$. For the Euler character $V$ is the space of differential forms on $M, Q=d+d^{*}$ is the sum of the exterior derivative operator and its adjoint, and $\Gamma=(-1)^{p}$ for $p$-forms, +1 for even-forms and -1 for odd-forms. The Euler character may be written as

$$
\chi(M)=\sum_{p} b_{p}
$$

where $b_{p}=\operatorname{dim}\left(M^{p}\right)$ is the $p^{\text {th }}$ Betti number, the dimension of the space $H^{p}$ of harmonic $p$-forms.

Formally, we may write the index (2.5) as

$$
I(Q)=\operatorname{Tr}(\Gamma)
$$

In general the right-hand side of (2.7) is not well-defined since it is the difference of two infinite quantities, the dimensions of $V_{ \pm}$. The vectors with positive $\lambda$ can be paired to give a canceling contribution to the trace, but (2.7) does not detect this. To make the right-hand side well-defined, we regulate it

$$
I(Q)=\operatorname{Tr}\left(\Gamma e^{-\beta \Delta}\right)
$$

If the spectrum of $\Delta$ is reasonably well-behaved, then the right-hand side of (2.8) will be finite and the contributions from vectors with nonzero $\lambda$ will cancel, leaving only the contribution of the zero-modes (2.5). This equation forms the basis for proving the Atiyah-Singer index theorem [1].

It remains only to show that this index is a topological invariant in the sense that it does not change under continuous changes of parameters in $Q$. This follows because the vectors with positive $\lambda$ occur in pairs. The most that can happen as the parameters in $Q$ are varied is that vectors can move towards or away from zero $\lambda$ in pairs. The net difference (2.5) is unchanged. The key technical assumption in this argument is that the spectrum of $\Delta$ be discrete.

The character-valued index is a generalization of this. Suppose that there is a group $G$ of symmetries of $V$ and $Q$; the group elements $g \in G$ commute with $Q$ and $\Gamma: g Q=Q g$ and $g \Gamma=\Gamma g$. The vectors of $V_{ \pm}$transform in complete representations of $G$ and, for nonzero $\lambda$, these representations come in pairs with opposite eigenvalues of $\Gamma$. We may then define the character-valued index as

$$
I_{g}(Q)=\operatorname{Tr}_{\mathrm{ker}\left(Q_{+}\right)}(g)-\operatorname{Tr}_{\mathrm{ker}\left(Q_{-}\right)}(g) \text {. }
$$

The ordinary index (2.5) is a special case of (2.9) when $g$ is the identity.

The most familiar example here is the Lefschetz number, which is the charactervalued index of the DeRham complex:

$$
L(g)=\sum_{p}(-1)^{p} \chi_{p}(g)
$$

where $\chi_{p}(g)=\operatorname{Tr}_{H^{p}}(g)$. The corresponding index theorem is just the Lefschetz fixed 
point theorem.

$$
L(g)=\chi\left(M_{g}\right)
$$

where $\chi\left(M_{g}\right)$ is the fixed-point space of $g$, the submanifold of $M$ left fixed by $g$.

Again, (2.9) is formally equivalent to $I_{g}(Q)=\operatorname{Tr}(\Gamma g)$, and when properly regulated, this gives

$$
I_{g}(Q)=\operatorname{Tr}\left(\Gamma g e^{-\beta \Delta}\right) .
$$

This formula will for the basis for the proof, given below, of the character-valued index theorem.

\section{Supersymmetric Quantum Mechanics}

To calculate these indices, we define a supersymmetric quantum mechanics model whose supersymmetry charge is the operator $Q$ in question. This method has been used before [6] to calculate the Atiyah-Singer index and to calculate a special case of the character-valued index [9].

We start with the superspace of $N=\frac{1}{2}$ supersymmetric quantum mechanics, defined by a single bosonic time coordinate $t$ and a single real fermionic coordinate $\theta$. A real scalar superfield has the form

$$
\Phi(t, \theta)=x(t)+i \theta \psi(t)
$$

where $x$ is a real scalar (commuting) field and $\psi$ is a real spinor (anticommuting) field. The corresponding free Lagrangian is

$$
L=\frac{1}{2} \int \mathrm{d} \theta D \Phi D^{2} \Phi=\frac{1}{2} \dot{x}^{2}+\frac{i}{2} \psi \dot{\psi}
$$

where $D=\partial / \partial \theta-i \theta(\partial / \partial t)$, is the superspace covariant derivative and $D^{2}=-i(\partial / \partial t)$.

The $N=\frac{1}{2}$ supersymmetric quantum mechanics of a particle on a manifold $M$, alternatively the supersymmetric nonlinear sigma model reduced to a single time dimension, is described by the modified lagrangian

$$
L=-\frac{1}{2} \int \mathrm{d} \theta g_{i j}(\Phi) D \Phi^{i} D^{2} \Phi^{j}=\frac{1}{2} g_{i j}\left(\dot{x}^{i} \dot{x}^{j}+i \psi^{i} D_{t} \psi^{j}\right),
$$

where $g_{i j}(\Phi)=g_{i j}(x)+i \theta \psi^{k} \partial_{k} g_{i j}(x)$ is defined by its Taylor series expansion in powers of $\theta$ and $D_{t} \psi^{i}=\dot{\psi}^{i}+\Gamma_{j k}^{i} \dot{x}^{j} \psi^{k}$ is the covariant time derivative for spinor fields. Here the $x^{i}$ are identified with the coordinates on a Riemannian manifold $M$ and the $\psi^{i}$ are identified with the tangent vector coordinates; $g_{i j}$ is the Riemannian metric and $\Gamma_{j k}^{i}$ is the associated Riemannian connection.

This system has a single real supersymmetry charge $Q=\partial / \partial \theta+i \theta(\partial / \partial t)$ satisfying the supersymmetry algebra $Q^{2}=i(\partial / \partial t)=H$. It acts on $\Phi^{i}$ as

$$
\xi Q \Phi^{i}=\xi\left(\frac{\partial}{\partial \theta}+i \theta \frac{\partial}{\partial t}\right)\left(x^{i}+i \theta \psi^{i}\right)=i \xi \psi^{i}-i \theta \xi \dot{x}^{i}
$$

leading to the transformation laws

$$
\partial_{\xi} x^{i}=i \xi \psi^{i}, \quad \partial_{\xi} \psi^{i}=-\xi \dot{x}^{i}
$$


where $\xi$ is an anticommuting $c$-number which parameterizes the transformation.

To quantize this system, we first change variables $\psi^{i}=e_{a}^{i} \psi^{a}$, where $\mathbf{e}_{a}=e_{a}^{i}\left(\partial / \partial x^{i}\right)$ is an orthonormal basis in the tangent space: $\mathbf{e}_{a} \cdot \mathbf{e}_{b}=\delta_{a b}$. After quantization the $\psi$ 's are identified with $\gamma$-matrices and this change of variables corresponds to the procedure in general relativity of defining the spin structure in terms of a vierbein $\gamma^{i}=e_{a}^{i} \gamma^{a}$. In terms of these variables, the lagrangian becomes

$$
L=\frac{1}{2} g_{i j} \dot{x}^{i} \dot{x}^{j}+\frac{i}{2} \delta_{a b} \psi^{a} D_{t} \psi^{b}
$$

where $D_{t} \psi^{a}=\dot{\psi}^{a}+\omega_{b i}^{a} \dot{x}^{i} \psi^{b}$ is the covariant derivative in terms of the new variables, and $\omega_{b i}^{a}=\mathbf{e}^{a}\left(\nabla_{i} \mathbf{e}_{b}\right)=e_{j}^{a} e_{b}^{k} \Gamma_{k i}^{j}+e_{j}^{a} \partial_{i} e_{b}^{j}$ is the connection in the orthonormal basis.

The quantization of this system is complicated slightly by the fact that the fermionic momentum and coordinate variable are identified,

$$
\Pi_{a}=\frac{\partial L}{\partial \dot{\psi}^{a}}=-\frac{i}{2} \delta_{a b} \psi^{b}
$$

This leads to a set of (fermionic) first-class constraints

$$
C_{a}=\Pi_{a}+\frac{i}{2} \delta_{a b} \psi^{b}=0
$$

and according to the quantization method of Dirac [10] the canonical (anti)commutation relations are modified by

$$
[A, B]_{ \pm} \rightarrow[A, B]_{ \pm}-\sum_{a b}\left[A, C_{a}\right]_{ \pm}\left(M^{-1}\right)^{a b}\left[C_{b}, B\right]_{ \pm},
$$

where $M_{a b}=\left[C_{a}, C_{b}\right]_{ \pm}$is the matrix of naive (anti)commutators between the constraints and $[,]_{ \pm}$denotes the commutator or anticommutator as appropriate. For the case at hand, this takes on a particularly simple form since $M_{a b}=\delta_{a b}$. The only relation which is changed in going to the Dirac bracket is the anticommutator $\left\{\psi^{a}, \psi^{b}\right\}=\delta^{a b}$

Using Noether's theorem the supersymmetry charge is determined to be

$$
Q=-\psi^{a} e_{a}^{i} g_{i j} \dot{x}^{j}=-\psi^{a} e_{a}^{i}\left(p_{i}-\frac{i}{2} \delta_{b d} \omega_{c i}^{d} \psi^{b} \psi^{c}\right) .
$$

Identifying the $\psi$ 's with Dirac $\gamma$-matrices $\psi^{a}=(1 / \sqrt{ } 2) \gamma^{a}$ and representing $p_{i}=$ $-i\left(\partial / \partial x^{i}\right)$, we see that the supersymmetry charge is just the Dirac operator on $M$ :

$$
\sqrt{ } 2 Q=i \bar{D}(g)=i \gamma^{a} e_{a}^{i} D_{i}
$$

where $D_{i}=\partial_{i}+\frac{1}{4} \delta_{a b} \omega_{c i}^{b} \gamma^{a} \gamma^{c}$ is the covariant derivative for tangent space spinors.

If the dimension $d=2 k$ of $M$ is even, then the Clifford algebra of the $\gamma$ matrices contains an independent matrix $\Gamma=i^{k} \gamma^{1} \gamma^{2} \cdots \gamma^{d}$ analogous to $\gamma^{5}$ in four dimensions, satisfying $\left\{\Gamma, \gamma^{a}\right\}=0$. In terms of $\psi^{i}$ this corresponds to the operator $(-1)^{F}$ which counts the fermion number modulo 2 and satisfies $(-1)^{F} \psi^{i}=-\psi^{i}(-1)^{F}$, as well as $Q(-1)^{F}=-(-1)^{F} Q$.

This allows us to separate the Hilbert space $V$ into bosonic and fermionic subspaces $V_{B}$ and $V_{F}$, which correspond to the spaces $V_{+}$and $V_{-}$of positive and 
negative chirality spinor fields on $M$, respectively. We may then identify the Dirac index $I(D)=\operatorname{Tr}(\Gamma)$ with the Witten index $\operatorname{Tr}(-1)^{F}$, although as above some regularization is required to make this well-defined, usually of the form $\operatorname{Tr}\left((-1)^{F} e^{-\beta H}\right)$. As before, this index is a topological invariant, invariant under continuous changes in parameters. In the next section we will calculate the regularized index $\operatorname{Tr}\left((-1)^{F} e^{-\beta H}\right)$ as a Euclidean functional integral and express it as a topological integral over $M$.

If $M$ has an isometry $b$, then we can calculate the character-valued index $I_{b}(D)=\operatorname{Tr}\left((-1)^{F} b e^{-\beta H}\right)$ as a functional integral with twisted boundary conditions:

$$
x(t=\beta)=b x(t=0), \quad \psi(t=\beta)=b \psi(t=0)
$$

This method was described in [9]. Unlike the techniques in [11 and 6] which rely on the existence of a Killing vector field, and therefore work only for continuous symmetries, this method applies to discrete symmetries as well.

If $d$ is odd, then $(-1)^{F}$ is a new operator which extends the naive Clifford algebra of Dirac matrices

$$
\gamma^{i} \rightarrow\left(\begin{array}{cc}
0 & \gamma^{i} \\
-\gamma^{i} & 0
\end{array}\right), \quad(-1)^{F} \rightarrow\left(\begin{array}{cc}
1 & 0 \\
0 & -1
\end{array}\right) .
$$

The two vector spaces $V_{B}$ and $V_{F}$ are then identical, and the index, as well as the character-valued index, vanishes.

3.1 Spinor Superfields. The Dirac index described above applies to a spinor interacting with a purely gravitational field. To calculate the Dirac index with gauge interactions as the Witten index of a supersymmetric theory, we need to introduce spinor superfields

$$
S(t, \theta)=\eta(t)+\theta \phi(t), \quad \bar{S}(t, \theta)=\bar{\eta}(t)+\theta \bar{\phi}(t) .
$$

The lagrangian for the noninteracting system is

$$
L^{\prime}=\int d \theta \bar{S} D S=i \bar{\eta} \dot{\eta}+\bar{\phi} \phi .
$$

Here $\phi$ is an auxiliary field, and can be eliminated through its equation of motion $\phi=0$.

To describe gauge interactions, we add a vector index $a$ which belongs to a representation $R$ of the gauge group and a gauge field $A_{i b}^{a}(x)$ on $M$, in addition to the original lagrangian (3.3). The fields $\eta^{a}$ and $\bar{\eta}_{a}$ will then be spinors with values in the vector bundle $E$ in the representation $R$ associated to the gauge potential $A$. The addition to the lagrangian which describes these interactions is

$$
L^{\prime}=\int d \theta \bar{S} D_{A} S=i \bar{\eta} D_{t} \eta+\bar{\phi} \phi+i \bar{\eta} \psi^{i} \psi^{j} \partial_{i} A_{j} \eta-\bar{\phi} \psi^{i} A_{i} \eta-\bar{\eta} \psi^{i} A_{i} \phi,
$$

where $D_{A}=D+i A_{i}(\Phi) D \Phi^{i}$ is the gauge covariant superspace derivative, $A_{i}(\Phi)=A_{i}(x)+i \theta \psi^{j} \partial_{j} A_{i}(x)$ is defined by its Taylor series expansion in $\theta$, and $\left(D_{i} \eta\right)^{a}=\dot{\eta}^{a}+i A_{i b}^{a} \psi^{i} \eta^{b}$ is the gauge covariant time derivative of $\eta$. $\phi$ remains an auxiliary field, and can be eliminated by its equation of motion $\phi^{a}=\psi^{i} A_{i b}^{a} \eta^{b}$ to give the lagrangian

$$
L^{\prime}=\bar{\eta} D_{t} \eta+\frac{i}{2} \bar{\eta} \psi^{i} \psi^{j} F_{i j} \eta
$$


The supersymmetry transformation is

$$
\xi Q S=\xi\left(\frac{\partial}{\partial \theta}+i \theta \frac{\partial}{\partial t}\right)(\eta+\theta \phi)=\xi \phi-i \theta \xi \dot{\eta}
$$

or

$$
\delta_{\xi} \eta=\xi \phi=\xi \psi^{i} A_{i} \eta, \quad \delta_{\xi} \bar{\eta}=\xi \bar{\phi}=\xi \bar{\eta} \psi^{i} A_{i} .
$$

Given this gauge coupling to the fermionic superfields, the supersymmetry charge is modified by the addition of a term

$$
Q \rightarrow Q+\bar{\eta} A_{i} \psi^{i} \eta
$$

After quantization, the supersymmetry charge is again identified with the Dirac operator, this time in the presence of the gauge connection $A$ and the metric $g$,

$$
\sqrt{ } 2 Q=i D(A, g) \text {. }
$$

Consider the Hilbert space $V_{1}$ consisting of states with a single $\eta$-fermion, i.e. states annihilated by all but one of the operators $\bar{\eta}^{a}$, but imposing no constraints on the number of $\psi$-fermions. These consist of spinor fields on $M$ with an extra index $a$ in the representation $R$ of the gauge group $G$ with connection $A$. On $V_{1}$, the supersymmetry charge is precisely the Dirac operator for spinors in this representation.

The index of this Dirac operator can then be identified with the Witten index restricted to the subspace $V_{1}$ :

$$
I(\mathbb{D}(A, g))=\operatorname{Tr}\left((-1)^{F} P_{1}\right)
$$

where $P_{1}$ is the projection operator onto the space $V_{1}$. Given an element $b$ of the (global) symmetry group $G$ of the vector bundle $E$, we may also define the charactervalued index $I_{b}(D(g, A))=\operatorname{Tr}_{\mathrm{V}_{+}}(b)-\operatorname{Tr}_{\mathrm{V}_{-}}(b)$, whose contribution comes entirely from the zero-modes as before, and this will correspond to the character-valued Witten index restricted to $V_{1}$. That is,

$$
I_{b}(\mathbb{D}(A, g))=\operatorname{Tr}\left((-1)^{F} b P_{1}\right) .
$$

The symmetry $b$ need not be merely an isometry of $M$, but may contain a compensating gauge transformation as well, i.e. an automorphism of the fibre $E_{x}$. This calculation was done in [9] for $N=1$ supersymmetric quantum mechanics, where the vector bundle $E$ is the same as the spinor bundle, and is generalized here for arbitrary vector bundles. In the next section, we will calculate the properly regulated version of the right-hand side of (3.22) as a Euclidean functional integral.

\section{Evaluation of the Functional Integral}

The regulated Witten index $\operatorname{Tr}\left((-1)^{F} e^{-\beta H}\right)$ can be calculated as the Euclidean functional integral with periodic boundary conditions for both fermions and bosons [12]. The ordinary Euclidean functional integral

$$
\operatorname{Tr}\left(e^{-\beta H}\right)=\int[d \phi] e^{-\int_{0}^{\beta} L_{E}(\phi)}
$$


is calculated by imposing periodic boundary conditions on all boson fields and antiperiodic boundary conditions on all fermion fields. Including the factor of $(-1)^{F}$ converts the boundary conditions for the fermions to periodic ones. It is not surprising that these boundary conditions are supersymmetric, i.e. the same for fermions and bosons.

The Euclidean lagrangian is derived from the real time one by replacing $H \rightarrow$ $-\partial / \partial t, Q \rightarrow \partial / \partial \theta-\theta(\partial / \partial t)$, and $D \rightarrow \partial / \partial \theta+\theta(\partial / \partial t)$, and by adjusting some phases in the lagrangian superfield

$$
\begin{aligned}
L_{E} & =\int d \theta\left(\frac{1}{2} g_{i j}(\Phi) D \Phi^{i} D^{2} \Phi^{j}-\bar{S}\left(D+i A_{i}(\Phi) D \Phi^{i}\right) S\right) \\
& =\frac{1}{2} g_{i j}\left(\dot{x}^{i} \dot{x}^{j}+\psi^{i} D_{t} \psi^{j}\right)+\bar{\eta} D_{t} \eta-\frac{i}{2} \bar{\eta} \psi^{i} \psi^{j} F_{i j} \eta
\end{aligned}
$$

The covariant time derivatives are as in Sect. 3.

Since the resulting functional integral is independent of $\beta$, it is convenient to take the limit $\beta \rightarrow 0$, when many higher-order interaction terms drop out from the action. As $\beta \rightarrow 0$ the penalty for time variation in the bosonic fields becomes larger and larger, and the nonconstant boson modes are confined to narrower and narrower ranges; they are then approximated better and better by tangent space variables. This allows the considerable simplification of describing their contributions to the functional integral as integrals over flat vector spaces. The two main problems in calculating the functional integral are to identify the leading terms as $\beta \rightarrow 0$ and to determine the proper integration measure.

We will first consider the problem without external gauge fields, and determine the normalization for the functional integration over the nonzero modes. This can be determined from the free Euclidean action, which reads

$$
L_{0}=\frac{1}{2} \sum_{i}\left(\left(\dot{x}^{i}\right)^{2}+\psi^{i} \dot{\psi}^{i}\right)
$$

Expanding the fields in Fourier components

$$
\begin{aligned}
x^{i} & =x_{0}^{i}+\sqrt{ } \beta \sum_{n=1}^{\infty}\left(a_{n}^{i} \cos (2 \pi n t / \beta)+b_{n}^{i} \sin (2 \pi n t / \beta)\right), \\
\psi^{i} & =\sqrt{\frac{i}{2 \pi \beta}} \psi_{0}^{i}+\sum_{n=0}^{\infty}\left(c_{n}^{i} \cos (2 \pi n t / \beta)+d_{n}^{i} \sin (2 \pi n t / \beta)\right),
\end{aligned}
$$

the action reads

$$
S_{E}=\sum_{i} \sum_{n=1}^{\infty}\left(\pi^{2} n^{2}\left(a_{n}^{i^{2}}+b_{n}^{i^{2}}\right)+\pi n c_{n}^{i} d_{n}^{i}\right)
$$

The factors of $\sqrt{\beta}$ and $\sqrt{i / 2 \pi \beta}$ have the effect of removing all $\beta$-dependence from the integration measure and setting the zero-mode measure to one.

Integrating over $a_{n}^{i}, b_{n}^{i}, c_{n}^{i}$, and $d_{n}^{i}$ for a fixed $n$ and $i$ produces a factor of $1 / n$, so inserting the proper normalization factor $N_{n}=n$ the functional measure can be written 


$$
[d x d \psi]=\prod_{i}\left(d x_{0}^{i} d \psi_{0}^{i} \prod_{n=1}^{\infty}\left(n d a_{n}^{i} d b_{n}^{i} d c_{n}^{i} d d_{n}^{i}\right)\right) .
$$

The next step is to introduce normal coordinates. Choosing a point $x \in M$ as the origin of coordinates, we may define a system of coordinates in which

$$
g_{i j}=\delta_{i j}-\frac{1}{3} R_{i k j l} x^{k} x^{l}+O\left(x^{3}\right)
$$

the procedure will be to integrate out first the variables $a_{n}^{i}, b_{n}^{i}, c_{n}^{i}$, and $d_{n}^{i}$, then the fermionic zero-modes $\psi_{0}^{i}$, all of which are tangent-space variables, and finally to integrate over the coordinates $x_{0}^{i}$. Since the functional integral is invariant under changes in coordinates, we may perform the tangent space integrations using normal coordinates centered at the corresponding point in $M$. This means that we may set $x_{0}^{i}=0$ when performing these integrations. The higher order terms in $x^{i}$ which appear in (4.7) will then drop off as powers of $\beta$ when $\beta \rightarrow 0$.

The interaction lagrangian in normal coordinates is

$$
\frac{1}{2} g_{i j} \Gamma_{k l}^{j} \psi^{i} \dot{x}^{k} \psi^{l}=\frac{1}{4} R_{i j k l} \psi^{i} \psi^{j} x^{k} \dot{x}^{l}+O\left(\psi^{2} x^{2} \dot{x}\right) .
$$

This gives a contribution to the action of order $\beta$ unless fermionic zero-modes are included, and the only surviving contribution in the limit $\beta \rightarrow 0$ is

$$
L_{I}=\frac{i}{8 \pi \beta} R_{i j k l} \psi_{0}^{i} \psi_{0}^{j} x^{k} \dot{x}^{l}=\frac{i}{4 \pi \beta} \Omega_{k l} x^{k} \dot{x}^{l},
$$

where $\Omega_{k l}=\frac{1}{2} R_{i j k l} \psi_{0}^{i} \psi_{0}^{j}$ can be interpreted as the curvature 2-form, provided we identify the fermionic zero modes with the basis 1 -forms: $\psi_{0}^{i} \leftrightarrow d x^{i}$. The contribution of $L_{I}$ to the action integral is

$$
\int_{0}^{\beta} L_{I} d t=\frac{i}{4 \pi} \Omega_{k l} \sum_{n=1}^{\infty} \pi n\left(a_{n}^{k} b_{n}^{l}-a_{n}^{l} b_{n}^{k}\right)
$$

The variables $c_{n}^{i}$ and $d_{n}^{i}$ have no interactions, and their functional integral remains trivial. To evaluate the remaining factors in the functional integral, we first specialize to the case where $\Omega$ can be put in skew-diagonal form:

$$
\Omega=\left[\begin{array}{cccc}
0 & \Omega_{1} & & \\
-\Omega_{1} & 0 & & \\
& & 0 & \Omega_{n} \\
& & -\Omega_{n} & 0
\end{array}\right]
$$

Considering only the first pair of coordinates and performing the integration for each $n$ by completing the squares, we arrive at the result

$$
\prod_{n>0} \frac{1}{1-\left(\frac{\Omega_{1}}{4 \pi^{2} n}\right)^{2}}=\frac{\Omega_{1} / 4 \pi}{\sinh \left(\Omega_{1} / 4 \pi\right)} .
$$

This has a power series expansion in $\Omega_{1}$ :

$$
\frac{\Omega_{1} / 4 \pi}{\sinh \left(\Omega_{1} / 4 \pi\right)}=1+\frac{1}{2}\left(\frac{\Omega_{1}}{4 \pi}\right)^{2}+\frac{7}{360}\left(\frac{\Omega_{1}}{4 \pi}\right)^{4}+\cdots
$$


with the product being the wedge product on forms. This series terminates because there are no higher forms than $d$-forms, or equivalently because of the Fermi statistics of the $\psi_{0}^{i}$. Integrating over the fermionic zero-modes projects out the coefficient of $\Pi \psi_{0}^{i}$ in the integrand, i.e. the top form (the $n$-form piece with the highest $n$ ), which is proportional to the volume form on $M$. For the form (4.11) of $\Omega$, the index is

$$
I(D(g))=\left.\int_{M} \prod_{n} \frac{\Omega_{n} / 4 \pi}{\sinh \left(\Omega_{n} / 4 \pi\right)}\right|_{\text {top form. }}
$$

In four dimensions this reduces to

$$
I(D(g))=-\frac{1}{96 \pi^{2}} \int_{M}\left(\Omega_{1}^{2}+\Omega_{2}^{2}\right)=\frac{1}{192 \pi^{2}} \int_{M} \operatorname{Tr} \Omega^{2} .
$$

This is the correct result even when $\Omega$ does not have the form (4.11), a result of the splitting principle [13].

Considering more general forms for $\Omega$, rescaled to absorb the overall normalization factor, the contribution to the action from the bosonic modes of fixed $n$ is

$$
S=\pi^{2} n^{2}\left(\begin{array}{ll}
a_{n}^{i} & b_{n}^{i}
\end{array}\right)\left(\begin{array}{cc}
\delta_{i j} & \frac{i \Omega_{i j}}{4 \pi^{2} n} \\
\frac{-i \Omega_{i j}}{4 \pi^{2} n} & \delta_{i j}
\end{array}\right)\left(\begin{array}{l}
a_{n}^{j} \\
b_{n}^{j}
\end{array}\right) .
$$

Integrating over these modes produces a factor of $\operatorname{det}^{-1 / 2}(1+A)$, where

$$
A=\frac{i}{4 \pi^{2} n}\left(\begin{array}{cc}
0 & \Omega \\
-\Omega & 0
\end{array}\right) .
$$

In any particular case, this integral can be expanded in powers of $A$ :

$$
\begin{aligned}
\operatorname{det}^{-1 / 2}(1+A) & =\exp \left(-\frac{1}{2} \operatorname{Tr} \log (1+A)\right)=\exp \frac{1}{2} \operatorname{Tr}\left(\frac{A^{2}}{2}+\frac{A^{4}}{4}+\cdots\right) \\
& =1+\frac{1}{4} \operatorname{Tr} A^{2}+\frac{1}{32}\left(\operatorname{Tr} A^{2}\right)^{2}+\frac{1}{8} \operatorname{Tr} A^{4}+\cdots \\
& =1+\frac{1}{32 \pi^{4} n^{2}} \operatorname{Tr} \Omega^{2}+\frac{1}{2048 \pi^{8} n^{4}}\left(\left(\operatorname{Tr} \Omega^{2}\right)^{2}+2 \operatorname{Tr} \Omega^{4}\right)+\cdots
\end{aligned}
$$

The important fact is that it is a sum of traces of products of curvature forms, i.e. a polynomial in Chern classes. The splitting principle then states that to calculate this polynomial, we may specialize to the case evaluated explicitly above, where the vector bundle in question splits into a sum of plane bundles.

In four dimensions, (4.18) yields a top-form coefficient of

$$
\sum_{n>0} \frac{1}{32 \pi^{4} n^{2}} \operatorname{Tr}\left(\Omega^{2}\right)=\frac{1}{192 \pi^{2}} \operatorname{Tr}\left(\Omega^{2}\right),
$$

which agrees with (4.15). In what follows, I will always use the splitting principle to calculate the functional integrals explicitly only in the relatively simple case where 
the vector bundles in question split into sums of line bundles, i.e. I will assume that complex vector bundles split into sums of complex line bundles. The more general expressions, when not explicitly written down, can be found by simply replacing sums over vector indices with traces, and products with determinants.

4.1 Including Gauge Interactions. There are two equivalent approaches to computing the Dirac index in the presence of external gauge fields from supersymmetric quantum mechanics. The first is to implement the projection onto $V_{1}$, the space of $\eta$ fermion number 1 , directly, i.e. to calculate the functional integral without assigning any dynamical significance to the $\eta$-fermions. We simply treat the $\eta$ fields as constants and take the trace of the action functional (the kinetic term vanishes) in the one $\eta$-fermion subspace $V_{1}$. The second is to assume dynamical $\eta$-fermions, i.e. to include a functional integral over $\eta$, and to project onto the subspace $V_{1}$. The second method is a bit more subtle, but provides special insight in the case the $\eta$-fermions are in a spinor representation $R$.

Adopting the analog of normal coordinates for the gauge connection

$$
A_{i}=-\frac{1}{2} F_{i j}^{0} x^{j}+O\left(x^{2}\right),
$$

where $F_{i j}^{0}=F_{i j}(x=0)$, sets the connection form $A$ to zero at the origin, and the connection becomes of order $x$. The relevant term in the lagrangian then becomes

$$
i \bar{\eta} A_{i} \dot{x}^{i} \eta=\frac{i}{2} \bar{\eta} F_{i j}^{0} \eta x^{i} \dot{x}^{j}+O\left(\bar{\eta} \eta x^{2} \dot{x}\right)
$$

and provides a contribution to the action of $\operatorname{order} \beta$, which drops out in the limit $\beta \rightarrow 0$. The only interaction term which does not disappear as $\beta \rightarrow 0$ is

$$
S_{I}=\frac{1}{2 \pi} \bar{\eta} \mathbf{F} \eta
$$

Where $\mathbf{F}=\frac{1}{2} F_{i j} \psi_{0}^{i} \psi_{0}^{j}$ is identified with the curvature 2-form of the connection $A$, provided we identify $d x^{i} \leftrightarrow \psi_{0}^{i}$ as before. Note that on subspace $V_{1}$, whose vectors carry a gauge index in the $R$ representation, the effective action acts as the matrix $\mathbf{F}$, i.e. $\bar{\eta} \mathbf{F} \eta \leftrightarrow \mathbf{F}$. The full functional integral then reads

$$
I(D(A, g))=\left.\int d V \operatorname{Tr}_{R} e^{-\mathrm{F} / 2 \pi} \prod_{n} \frac{\Omega_{n} / 4 \pi}{\sinh \left(\Omega_{n} / 4 \pi\right)}\right|_{\text {top form. }}
$$

This agrees with the standard result for the Dirac index on a two-dimensional manifold

$$
I(D(A, g))=-\frac{1}{2 \pi} \int_{M} \operatorname{Tr}_{R} \mathbf{F}
$$

and on a four-dimensional manifold

$$
I(D(A, g))=\frac{1}{8 \pi^{2}} \int_{M} \operatorname{Tr}_{R} \mathbf{F}^{2}+\frac{\operatorname{dim}(R)}{192 \pi^{2}} \int d V \operatorname{Tr} \Omega^{2}
$$

Proceeding with the second method for calculating the Dirac index, we need to do two things. First, we need to expand the $\eta$ fields in Fourier modes and determine 
the proper integration measure. Second, we need to write down the projection operator $P_{1}$ onto the subspace $V_{1}$ with a single $\eta$-fermion.

We expand the fields $\eta$ and $\bar{\eta}$ in Fourier modes as

$$
\eta^{a}=\sum_{n=-\infty}^{\infty} \eta_{n}^{a} e^{2 \pi \mathrm{in} t / \beta}, \quad \bar{\eta}_{a}=\sum_{n=-\infty}^{\infty} \bar{\eta}_{n a} e^{-2 \pi \mathrm{int} / \beta}
$$

The free action for these modes is

$$
\int_{0}^{\beta} d t \bar{\eta}_{a} \dot{\eta}^{a}=\sum_{n} 2 \pi i n \bar{\eta}_{a n} \eta_{n}^{a}
$$

and the integral over modes with fixed $n$ and $a$ gives

$$
\int d \bar{\eta}_{a n} d \eta_{n}^{a} e^{-2 \pi i n \bar{\eta}_{a n} \eta_{n}^{a}}=2 \pi i n,
$$

so with the proper normalization factor of $1 / 2 \pi i n$, the functional measure for the $\eta$ fermions becomes

$$
[d \bar{\eta} d \eta]=\prod_{a}\left(-d \bar{\eta}_{a 0} d \eta_{0}^{a} \prod_{n \neq 0}\left(\frac{1}{2 \pi i n} d \bar{\eta}_{a n} d \eta_{n}^{a}\right)\right)
$$

The $\eta$-fermion number operator is

$$
N_{\eta}=\sum \bar{\eta}_{a n} \eta_{n}^{a}+c
$$

where $c=\operatorname{dim}(R) / 2$ is a normal ordering constant. The projection operator $P_{1}$ for $\eta$ fermion number one is

$$
P_{1}=N \int_{0}^{2 \pi} \frac{d \lambda}{2 \pi} e^{i \lambda\left(N_{\eta}-1\right)}
$$

where $N$ is a normalization factor to be determined below. The calculation proceeds as follows: First we perform the functional integration of the $\eta$-fermions. Then we integrate over $\lambda$ to project onto the $N_{\eta}=1$ sector, identifying the proper normalization factor $N$. Finally, we will integrate out the $\psi$-fermions and the nonzero modes of $x$, leaving us with an integral over $M$ of Chern classes of both the spin bundle and the gauge vector bundle.

Again, the interaction term which will survive as $\beta \rightarrow \infty$ is

$$
L_{I}=\frac{1}{2 \pi \beta} \bar{\eta}_{a} \mathbf{F}_{b}^{a} \eta^{b}
$$

First we assume $\mathbf{F}_{b}^{a}=\mathbf{F}_{a} \delta_{b}^{a}$ (the splitting principle) and write the effective action for the $\eta$-fermions, including the term $-i \lambda\left(N_{\eta}-c\right)$ which comes from $P_{1}$, as

$$
S_{\mathrm{eff}}(\eta)=\sum_{a, n} \bar{\eta}_{a n}\left(2 \pi i n-i \lambda+\mathbf{F}_{a} / 2 \pi\right) \eta_{n}^{a}
$$

Integrating and reinserting the factor of $e^{i c \lambda}$, this yields

$$
\int[d \bar{\eta} d \eta] e^{-S_{\text {eff }(\eta)}}=e^{i c \lambda} \prod_{a} 2 i \sin \left(\frac{\lambda+i \mathbf{F}_{a} / 2 \pi}{2}\right)=e^{\operatorname{Tr} \mathbf{F} / 4 \pi} \prod_{a}\left(1-e^{i\left(\lambda+i \mathbf{F}_{a} / 2 \pi\right)}\right) .
$$


The terms proportional to $e^{i \lambda n}$ correspond to the sector with $n \eta$-fermions. Note that each factor of $e^{i \lambda}$ carries an extra minus sign, indicating that adding an $\eta$-fermion changes the sign of $(-1)^{F}$, as expected. The sector with no $\eta$-fermions should give a factor of one in order to reproduce the result (4.14), leading us to identify the normalization factor

$$
N=e^{-\operatorname{Tr} \mathbf{F} / 4 \pi} .
$$

Projecting onto the subspace $V_{1}$ then reproduces the result $\operatorname{Tr}_{R} e^{-\mathbf{F} / 2 \pi}$ in (4.23) (with an extra minus sign from the fermion number of the $\eta$ fields, which was ignored above).

4.2 The Character-Valued Index. To calculate the character-valued index, we first specialize to the case where there are no background gauge fields. Inserting the factor of $b$ in the functional integral amounts to integrating over configurations with twisted boundary conditions rather than simple periodic ones.

As $\beta \rightarrow 0$, paths which go from a point $x$ to its image $b x$ under $b$ will have their contribution to the functional integral suppressed by an exponential factor of $e^{-d^{2}(x, b x) / \beta}$, where $d(x, b x)$ is the distance between $x$ and $b x$, unless this distance is zero, i.e. unless $x=b x$ is a fixed point of $b$. The functional integral is dominated by the contribution from the fixed point space $M_{b}$.

The neighborhood of $M_{b}$ in $M$ is well approximated by the normal bundle $N_{M}\left(M_{b}\right)$ to $M_{b}$ in $M$. On this normal bundle, $b$ acts as a simple rotation, and can be block-diagonalized to rotate pairs of coordinates $x^{r}$ and $\bar{x}^{r}$ through an angle $\theta_{r}$ :

$$
b:\left(\begin{array}{l}
x^{r} \\
x^{r}
\end{array}\right) \rightarrow\left(\begin{array}{cc}
\cos \theta_{r} & -\sin \theta_{r} \\
\sin \theta_{r} & \cos \theta_{r}
\end{array}\right)\left(\begin{array}{l}
x^{r} \\
x^{\bar{r}}
\end{array}\right) .
$$

In terms of the complex variable $X^{r}=x^{r}+i x^{\bar{r}}$, this transformation can be written

$$
b: X^{r} \mapsto e^{i \theta_{r}} X^{r} .
$$

The transformation of the fermionic variables, which describe tangent space coordinates, is identical:

$$
b:\left(\begin{array}{l}
\psi^{r} \\
\psi^{\tilde{r}}
\end{array}\right) \mapsto\left(\begin{array}{cc}
\cos \theta_{r} & -\sin \theta_{r} \\
\sin \theta_{r} & \cos \theta_{r}
\end{array}\right)\left(\begin{array}{l}
\psi^{r} \\
\psi^{\tilde{r}}
\end{array}\right) .
$$

In terms of complex fermionic variables $\Psi^{r}=\psi^{r}+i \psi^{\bar{r}}$ this becomes

$$
\text { b: } \Psi^{r} \mapsto e^{i \theta_{r}} \Psi^{r} .
$$

For a discrete symmetry $b$ there is some integer $n$ such that $b^{n}=1$, and we must have $n \theta_{r}=2 \pi m$ for some integer $m$. If $b=e^{i \lambda T}$ belongs to a $U(1)$ group generated by $T$ with $e^{2 \pi i T}=1$, then we must have $\theta_{r}=m \lambda$ for some integer $m$.

Imposing the twisted boundary conditions

$$
X^{r}(\beta)=e^{i \theta_{r}} X^{r}(0), \Psi^{r}(\beta)=e^{i \theta_{r}} \Psi^{r}(0)
$$

simply shifts the Fourier expansion of these variables

$$
X^{r}=\sqrt{ } \beta \sum_{m=-\infty}^{\infty}\left(a_{m}^{r}+i b_{m}^{r}\right) e^{i\left(2 \pi n+\theta_{r}\right) t / \beta}, \Psi^{r}=\sum_{m=-\infty}^{\infty}\left(c_{m}^{r}+i d_{m}^{r}\right) e^{i\left(2 \pi n+\theta_{r}\right) t / \beta} .
$$


The kinetic piece of the action then becomes

$$
\begin{aligned}
& \frac{1}{2} \int_{0}^{\beta} d t\left(\dot{x}^{r^{2}}+\dot{x}^{\bar{r}^{2}}\right)=\frac{1}{2} \sum_{n}\left(2 \pi n+\theta_{r}\right)^{2}\left(a_{n}^{r^{2}}+b_{n}^{r^{2}}\right), \\
& \frac{1}{2} \int_{0}^{\beta} d t\left(\psi^{r} \dot{\psi}^{r}+\psi^{\bar{r}} \dot{\psi}^{r}\right)=-\sum_{n}\left(2 \pi n+\theta_{r}\right) c_{n}^{r} d_{n}^{r},
\end{aligned}
$$

and the properly normalized functional measure reads

$$
\left[d x^{r} d \psi^{r}\right]=-\frac{1}{2 \pi} d a_{0}^{r} d b_{0}^{r} d c_{0}^{r} d d_{0}^{r} \prod_{n \neq 0}\left(-n d a_{n}^{r} d b_{n}^{r} d c_{n}^{r} d d_{n}^{r}\right)
$$

In addition to the interaction terms for the base-space variables, there is an additional contribution from the normal-bundle variables which remains important as $\beta \rightarrow 0$. It is

$$
L_{I}=\frac{i}{8 \pi \beta} \psi_{0}^{i} \psi_{0}^{j}\left(R_{i j r r} x^{r} \dot{x}^{\bar{r}}+R_{i j r r} x^{\bar{r}} \dot{x}^{r}\right)=\frac{i}{4 \pi \beta} \Omega_{r}\left(x^{r} \dot{x}^{\bar{r}}-x^{\bar{r}} \dot{x}^{r}\right),
$$

where $\Omega_{r}=\frac{1}{2} \psi_{0}^{i} \psi_{0}^{j} R_{i j r r}$ is the curvature 2-form on the normal bundle, specialized to the case where the normal bundle can be split into plane bundles. The contribution to the action of this interaction term is

$$
\frac{i}{4 \pi} \int_{0}^{\beta} \Omega_{r}\left(x^{r} \dot{x}^{\bar{r}}-x^{\bar{r}} \dot{x}^{r}\right)=\frac{i \Omega_{r}}{4 \pi} \sum_{n}\left(2 \pi n+\theta_{r}\right)\left(a_{n}^{r^{2}}+b_{n}^{r^{2}}\right) .
$$

Including the integral (4.14) over the base space, the functional integral becomes

$$
I_{b}(D(g))=\left.\int_{M_{b}} \prod_{n} \frac{\Omega_{n} / 4 \pi}{\sinh \left(\Omega_{n} / 4 \pi\right)} \prod_{r} \frac{1}{2 i \sin \left(\bar{\theta}_{r} / 2\right)}\right|_{\text {top form. }}
$$

where $\bar{\theta}_{r}=\theta_{r}+(i / 2 \pi) \Omega_{r}$. In the special case where there are only isolated fixed points, this reproduces the standard expression [14]

$$
I_{b}(D(g))=\sum_{\text {fixed pts. }} \prod_{r} \frac{1}{2 \sin \left(\theta_{r} / 2\right)} .
$$

4.3 The Character-Valued Index with Gauge Interactions. Since $\eta^{a}$ and $\bar{\eta}_{a}$ are vector space coordinates, $b$ will simply rotate the $\eta$-fermions among themselves. Since these are complex variables, a rotation here is equivalent to multiplication by a phase:

$$
b: \eta^{a} \mapsto e^{i \theta_{a}} \eta^{a}, \quad \bar{\eta}_{a} \mapsto e^{-i \theta_{a}} \bar{\eta}_{a} .
$$

This first method for dealing with the $\eta$-fermions allows the straightforward generalization of simply inserting the operator $b$ into the group trace. Including the factors in (4.46) this gives

$$
\begin{aligned}
I_{b}(\mathbb{D}(g, A)) & =\int_{M_{b}} \operatorname{Tr}_{R}\left(b e^{-\mathbf{F} / 2 \pi}\right) \prod \frac{\Omega_{n} / 4 \pi}{\sinh \left(\Omega_{n} / 4 \pi\right)} \prod_{r} \frac{1}{2 i \sin \left(\theta_{r} / 2\right)} \\
& =\int_{M_{b}} \sum_{a} e^{-i \bar{\theta}_{a}} \prod_{n} \frac{\Omega_{n} / 4 \pi}{\sinh \left(\Omega_{n} / 4 \pi\right)} \prod_{r} \frac{1}{2 i \sin \left(\bar{\theta}_{r} / 2\right)},
\end{aligned}
$$


where $\bar{\theta}_{a}=\theta_{a}-(i / 2 \pi) \mathbf{F}_{a}$ and we have assumed that the gauge vector bundle splits into complex line bundles as indicated.

The second method for dealing with $\eta$-fermions produces the same result. Inserting $b$ into the functional integral imposes twisted boundary conditions on the $\eta$-fermions:

$$
\eta^{a}(\beta)=e^{i \theta_{a}} \eta^{a}(0), \quad \bar{\eta}_{a}(\beta)=e^{-i \theta_{a}} \bar{\eta}_{a}(0)
$$

and shifts their Fourier expansions to

$$
\eta^{a}=\sum_{n} \eta_{n}^{a} e^{i\left(2 \pi n+\theta_{a}\right) t / \beta}, \quad \bar{\eta}_{a}=\sum_{n} \bar{\eta}_{a n} e^{-i\left(2 \pi n+\theta_{a}\right) t / \beta} .
$$

The kinetic term in the action for $\eta$ then reads

$$
\int_{0}^{\beta} d t \bar{\eta}_{a} \dot{\eta}^{a}=i \sum_{n}\left(2 \pi n+\theta_{a}\right) \bar{\eta}_{a n} \eta_{n}^{a}
$$

Again, the only interaction piece which does not vanish as $\beta \rightarrow 0$ is

$$
L_{I}=\frac{1}{2 \pi \beta} \bar{\eta}_{a} \mathbf{F}_{b}^{a} \eta^{b}
$$

The resulting $\eta$-fermion effective action is

$$
S_{\mathrm{eff}}(\eta)=\sum_{a, n} \bar{\eta}_{n}^{a}\left(2 \pi i n+i \theta_{a}+\mathbf{F}_{a} / 2 \pi-i \lambda\right) \eta_{a n}
$$

The net effect is to replace $-\mathbf{F}_{a} / 2 \pi$ with $-i \theta_{a}-\mathbf{F}_{a} / 2 \pi=-i \bar{\theta}_{a}$, which transforms (4.23) into (4.49) as expected

4.4 Spinor Representations, Euler Character, Hirzebruch Signature. In the case where $R$ is a spinor representation of $S O(2 N)$ we may calculate the index and the character-valued index as in the previous section, but in this case there is another, more interesting possibility. Instead of using fermions in the representation $R$, which leads to the indices (4.23) and (4.49), we may use fermions in the Clifford algebra of $R$. To distinguish from the previous case, I will denote such fermions by $\gamma$, indicating their relationship with the Dirac $\gamma$-matrices. The resulting indices will differ from the previous ones in a way which corresponds, in the notation of Sect. 3, to keeping the same operator $Q$, but choosing a different $\Gamma$.

In the case where $R$ is the same representation as that of the tangent spinors these indices correspond to familiar topological invariants associated with the deRham complex on $K$. The index defined in the previous sections corresponds to the Hirzebruch signature and its character-valued generalization, the Atiyah-Bott signature [15]. The index coming from fermions in the Clifford algebra will lead to the Euler character and the Lefschetz number.

The free Euclidean lagrangian for $\gamma$-fermions is

$$
L=\frac{1}{2} \sum_{i} \gamma^{i} \dot{\gamma}^{i}
$$

The $\gamma$-fermions, like $\psi$-fermions, are real. To quantize them, it is useful to transform them into a complex basis $\eta^{a}=1 / \sqrt{ } 2\left(\gamma^{2 a-1}+i \gamma^{2 a}\right)$ and $\bar{\eta}_{a}=1 / \sqrt{ } 2\left(\gamma^{2 a-1}-i \gamma^{2 a}\right)$. Up 
to a total derivative, the lagrangian takes the form

$$
L=\sum_{a} \bar{\eta}_{a} \dot{\eta}^{a}
$$

The representation $R$ can then be identified with the Fock space generated by the operators $\bar{\eta}_{a}$, i.e. with antisymmetric products of the complex vector representation generated by the $\eta_{a}$. In calculating the Witten index, we will be assigning fermion number to the vectors in this Fock space according to the number of $\eta$-fermions they contain $^{1}$. In Eqs. (4.23) and (4.49), on the other hand, all the vectors in the representation $R$ are assigned fermion number one. This construction therefore results in a different index from the one calculated above. In the case where the spinors in $R$ are tangent spinors on $M$, the resulting index will be the Euler character, where the earlier construction would have given the Hirzebruch signature.

When using Clifford-algebra fermions for calculating the index, the projection onto the one-fermion subspace is no longer necessary. The resulting functional integral is similar to (4.34) but without the variable $\lambda$, i.e.

$$
I(D(A, g))=\int_{M} \prod_{n} \frac{\Omega_{n} / 4 \pi}{\sinh \left(\Omega_{n} / 4 \pi\right)} \prod_{a} 2 \sinh \left(F_{a} / 4 \pi\right)
$$

for the ordinary index, or

$$
I_{b}(D(A, g))=\int_{M_{b}} \prod_{n} \frac{\Omega_{n} / 4 \pi}{\sinh \left(\Omega_{n} / 4 \pi\right)} \prod_{r} \frac{1}{2 i \sin \left(\bar{\theta}_{r} / 2\right)} \prod_{a} 2 i \sin \left(\bar{\theta}_{a} / 2\right)
$$

for the character-valued index.

For tangent spinors $\gamma^{i}$ the connection is precisely the spin connection and the curvature is the Riemann curvature. The interaction lagrangian is

$$
L_{I}=\frac{i}{2 \pi \beta} R_{i j k l} \psi_{0}^{i} \psi_{0}^{j} \gamma^{k} \gamma^{i}=\frac{i}{4 \pi \beta} \Omega_{k l} \gamma^{k} \gamma^{i}
$$

When the curvature 2-form has the skew-diagonal form (4.11), this reads

$$
L_{I}=\sum_{a} \frac{i \Omega_{a}}{2 \pi \beta} \gamma^{2 a-1} \gamma^{2 a}=-\sum_{a} \frac{\Omega_{a}}{2 \pi \beta} \bar{\eta}_{a} \eta^{a} .
$$

The eigenvalues $\mathbf{F}_{a}$ correspond to the skew-eigenvalues $-\Omega_{a}$. The result is a remarkable cancellation in (4.57), leaving

$$
I(D(A, g))=\int_{M} \prod_{n}\left(-\frac{\Omega_{n}}{2 \pi}\right)=\chi(M),
$$

where $\chi(M)$ is the Euler character of $M$. For the character-valued index, the cancellation in (4.58) is equally impressive, leaving

$$
I_{b}(\mathbb{D}(A, g))=\int_{M_{b}} \prod_{n}\left(-\frac{\Omega_{n}}{2 \pi}\right)=\chi\left(M_{b}\right) .
$$

1 The separation into $\eta^{a}$ and $\bar{\eta}_{a}$ may be possible only locally and not globally. Fermion number may not be well-defined in general, but $(-1)^{F}$ is 
The reason for this cancellation is that the lagrangian in question has a higher supersymmetry than before, $N=1$ instead of $N=\frac{1}{2}$. The fields, relabeled as

$$
\psi^{i} \rightarrow \psi_{1}^{i}, \quad \gamma^{i} \rightarrow \psi_{2}^{i}
$$

and including the auxilliary fields $F^{i}$ associated to $\gamma^{i}$ fit into a single $N=1$ superfield:

$$
\Phi^{i}=x^{i}+i \theta_{1} \psi_{1}^{i}+i \theta_{2} \psi_{2}^{i}+i \theta_{1} \theta_{2} F^{i}
$$

The euclidean lagrangian for these fields is

$$
L=\frac{1}{2} g_{i j}\left(\dot{x}^{i} \dot{x}^{j}+\psi_{1}^{i} D_{t} \psi_{1}^{j}+\psi_{2}^{i} D_{t} \psi_{2}^{j}\right)+\frac{1}{4} R_{i j k l} \psi_{1}^{i} \psi_{1}^{j} \psi_{2}^{k} \psi_{2}^{l}
$$

This model has the supersymmetry

$$
\delta x^{i}=\xi^{*} \Psi^{i}, \quad \delta \Psi^{i}=-i \xi \dot{x}^{i}-\Gamma_{j k}^{i} \xi \Psi^{j *} \Psi^{k},
$$

where $\xi$ is a complex fermionic parameter and $\Psi^{i}=\psi_{1}^{i}+i \psi_{2}^{i}$.

Upon quantization, the states $|\omega\rangle$ of the theory are identified with the differential forms $\omega$ on $M$, the fermionic variables with the basis 1 -forms $\Psi^{i}|\omega\rangle=\left|d x^{i} \wedge \omega\right\rangle$ and the supersymmetry charge with the exterior derivative $Q|\omega\rangle=|d \omega\rangle$. The operator $(-1)^{F}$ is simply $(-1)^{p}$ on $p$-forms. The hamiltonian is the laplacian $\Delta=Q Q^{*}+Q^{*} Q=d d^{*}+d^{*} d$, so the zero-energy states are the harmonic forms, and the corresponding index is just the Euler character

$$
\operatorname{Tr}\left((-1)^{F} e^{-\beta H}\right)=\sum_{p}(-1)^{p} b_{p}=\chi(M),
$$

where $b_{p}=\operatorname{dim}\left(H^{p}\right)$ is the $p^{t h}$ Betti number, the dimension of the space $H^{p}$ of harmonic $p$-forms.

The second result above can be restated in the language of forms as

$$
\operatorname{Tr}\left((-1)^{F} b e^{-\beta H}\right)=\sum_{p} \chi_{p}(b)=\chi\left(M_{b}\right),
$$

where $\chi_{p}(b)=\operatorname{Tr}_{H^{p}}(b)$ is the trace of $b$ in the space of harmonic $p$-forms. This result (4.68) is the Lefschetz fixed point theorem, a special case of the character-valued index theorem.

The earlier indices (4.23) and (4.49) defined when the fermionic representation is the one-particle space generated by fermions in that representation, rather than by a Clifford algebra, is also familiar in the special case of tangent spinors. In this case, the states in the "gauge" fermion vector space are all assigned fermion number one. This is as if $\psi_{1}^{i}$ were given fermion number one and $\psi_{2}^{i}$ fermion number zero, and corresponds to the chiral symmetry of the lagrangian (4.59)

$$
Q_{5}: \Psi^{i} \rightarrow \Psi^{i *}
$$

In the language of forms, this interchanges $p$-forms with $(n-p)$-forms, and implements Hodge duality $Q_{5}|\omega\rangle=|* \omega\rangle$.

The corresponding index using $Q_{5}$ in the place of $(-1)^{p}$ is the Hirzebruch signature, which (4.23) gives as

$$
I(D(A, g))=\int_{M} \prod_{n} \frac{\Omega_{n} / 4 \pi}{\tanh \left(\Omega_{n} / 4 \pi\right)},
$$


the Hirzebruch signature the theorem. The corresponding character-valued index (4.49) is

$$
I_{b}(D(A, g))=\int_{M_{b}} \prod_{n} \frac{\Omega_{n} / 4 \pi}{\tanh \left(\Omega_{n} / 4 \pi\right)} \prod_{r} \cot \left(\bar{\theta}_{r} / 2\right)
$$

the Atiyah-Bott fixed point theorem [15].

\section{Conclusions}

When a manifold $M$ has a nontrivial group of automorphisms which extend to automorphisms of a vector bundle $E$ over $M$, the Atiyah-Singer index theorem has a natural generalization, the character-valued index theorem. This index theorem describes how the zero-modes of the Dirac operator transform under this automorphism group. This theorem has an important physical application to theories in more than four spacetime dimensions, where it describes the transformation properties of the massless fields, which in turn restrict the possible interactions in the low-energy field theory.

Acknowledgements. I would like to thank Edward Witten for suggesting this work, and Emil Martinec for a helpful discussion of some technical points.

\section{References}

1. Atiyah, M. F., Singer, I. M.: Bull. Am. Math. Soc., 69, 422 (1963); Atiyah, M. F., Singer, I. M.: Ann. Math. 87, 484 (1968); ibid., 546

2. Bell, J. S., Jackiw, R.: Phys. Lett. 59B, 85 (1969); Adler, S. L.: Phys. Rev. 117, 2426 (1969)

3. Hooft, G.'t: Symmetry breaking through Bell-Jackiw anomalies. Phys. Rev. Lett. 37, 8 (1976); Callen, C. G. et al.: The structure of the gauge theory vacuum. Phys. Lett. 63B, 334 (1976); Jackiw, R., Rebbi, C.: Vacuum periodicity in a Yang-Mills quantum theory. Phys. Rev. Lett. 37, 172 (1976)

4. Atiyah, M. F., Singer, I. M.: Proc. Nat. Acad. Sci. USA 81, 409 (1984); Alvarez-Gaumé, L., Ginsparg, P.: The topological meaning of non-abelian anomalies. Nucl. Phys. B243, 449 (1984)

5. Witten, E.: Constrains on supersymmetry breaking. Nucl. Phys. B202, 253 (1982)

6. Alvarez-Gaumé, L.: Supersymmetry and the Atiyah-Singer index theorem. Commun. Math. Phys. 90, 161 (1983); Alvarez-Gaumé, L.: A note on the Atiyah-Singer index theorem. J. Phys. A16, 4177 (1983); Friedan, D., Windey, P.: Supersymmetric derivation of the Atiyah-Singer index and the chiral anomaly. Nucl. Phys. B235, 395 (1984)

7. Atiyah, M. F., Bott, R.: Ann. Math. 86, 374 (1967); ibid., 88, 451 (1968); Atiyah, M. F., Segal, G. B.: Ann. Math. 86, 531 (1968); Atiyah, M. F., Singer, I. M.: Ann. Math. 86, 546 (1968)

8. Witten, E.: In: Shelter Island II: Proceedings of the 1983 shelter Island conference on quantum field theory and the fundamental problems of physics. Khuri, N. et al. (eds.) Cambridge, MA: MIT Press 1985

9. Goodman, M. W., Witten, E.: Global symmetries in four and higher dimensions. Nucl. Phys. B (to appear)

10. Dirac, P. A. M.: Can. J. Math. 2, 129 (1950)

11. Witten, E.: J. Math. Phys. 17, 661 (1982)

12. Cecotti, S., Girardello, L.: Functional measure, topology and dynamical supersymmetric breaking. Phys. Lett. 110B, 39 (1982)

13. Hirzebruch, F.: Topological Methods in Algebraic Geometry. Berlin, Heidelberg, New York: Springer 1966; Bott, R., Tu, L. W.: Differential forms in algebraic topology. Berlin, Heidelberg, New York: Springer. 1982. 
14. Eguchi, T., et al.: Gravitation, gauge theories and differential geometry. Phys. Rep. 66, 213 (1980) 15. Atiyah, M. F., Bott, R.: Ann. Math. 88, 451 (1968)

Communicated by A. Jaffe

Received May 29, 1986; in revised form July 1, 1986 
